electronic devices, while the group at UIUC are using their approach to synthesize magnetic nanocomposites for permanent magnet applications.

\section{More Accurate Atomic Clocks May Be Enabled by Optical Frequency Combs from Femtosecond Lasers}

Physicist Scott Diddams of the National Institute of Standards and Technology (NIST) and visiting scientist Thomas Udem of the Max Planck Institute for Quantum Optics are part of the NIST team that has developed an optical atomic clock with the potential to be 1000 times more accurate than currently available atomic clocks.

Current atomic clocks operate at microwave frequencies to measure the oscillations of cesium atoms, about 9 billion per second, accurately. The new optical clock operates at a much higher optical frequency, about 100,000 times higher than a microwave clock. Building a clock based on such a high-frequency transition was previously impractical because it requires both "capturing" the ion and holding it very still to get accurate readings, and having a mechanism that can "count" the ticks accurately at such a high frequency. To accomplish this, the scientists use lasers that can deliver pulses of laser light that last just a few femtoseconds. Such lasers typically have their longitudinal optical modes locked to produce a stable optical frequency comb.

As reported in the August 3 issue of Science, the clock combines recent advances in three areas of research: the trapping and cooling of atoms and ions with lasers, frequency-stabilized lasers, and an optical frequency "comb" that combines a femtosecond laser with nonlinear optical fibers to provide a simple, direct, and exact linkage between microwave and optical frequencies. It is the last development that enables the device to count individual cycles of such a high frequency without skipping any, and thus permits the readout of time.
The femtosecond laser-based clockwork divides the optical frequency of the clock's "pendulum" into a countable microwave frequency. A single mercury ion serves as the reference for the optical atomic clock, providing long-term stability and accuracy.

\section{Strontium Naturally Tags Salmon from Specific Geologic Areas}

Researchers Brian Kennedy, Andrea Klaue, and Joel Blum at the University of Michigan and Carol Folt of Dartmouth College have found that the element strontium, relatively common in bedrock beneath streams, accumulates in the bony tissues of Atlantic salmon and leaves a specific chemical signature, depending on the geology of the watershed in which the fish are living. By taking advantage of the natural variation in strontium isotopes, scientists now can differentiate fish from specific geologic areas without having to use a human-made marker previously attached to a fish.
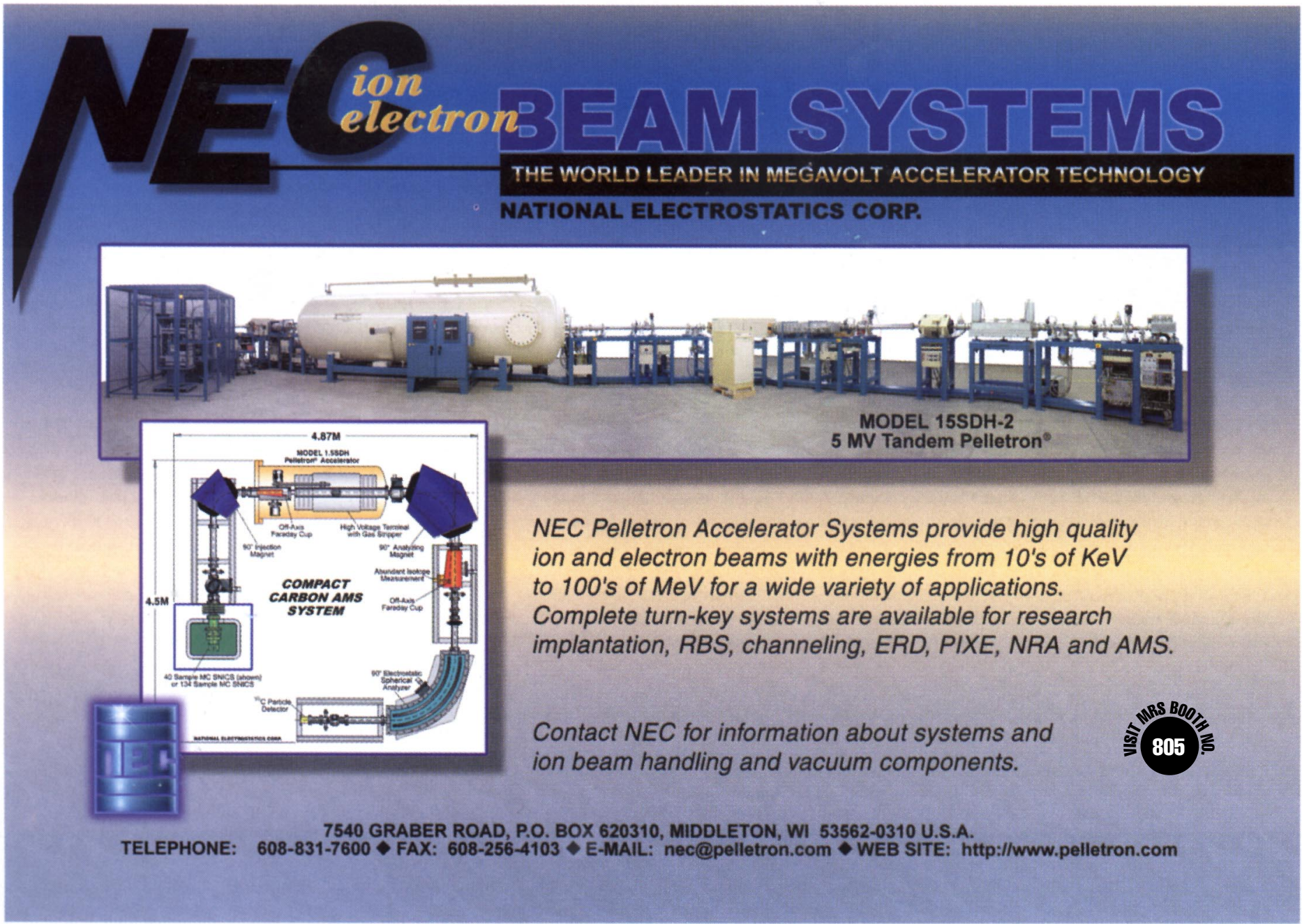